\title{
Diabetes drug could lead to longer life
}

Previous studies in humans have credited a diabetes drug called metformin with reducing the risks of developing pancreatic cancer and breast cancer. Another study found that ovarian cancer patients taking metformin for their diabetes lived longer than those who did not take the drug. Given these findings, Rafael de Cabo of the National Institute on Aging (Baltimore, MD) thought that metformin might be a good candidate for studying ways to improve aging and lengthen lifespan.

When de Cabo and his team gave a small dose of metformin to middle-aged mice, the drug boosted their health and extended their lives (Nat. Commun. published online 30 July 2013; doi:10.1038/ncomm3192). The scientists tested two doses of the drug. Mice that were given $0.1 \%$ metformin mixed into their diets lived almost $6 \%$ longer than untreated mice: the treated mice lived 160 weeks, while those not given the drug lived about 150 weeks. But when mice were given $1 \%$ metformin in their diets, their lifespans were shortened by about $14 \%$ compared to untreated mice. The latter result was likely due to the toxicity of the high dose, which induced kidney failure in the mice.

Aging is a driving force behind metabolic syndrome, which is a predisposing condition for type 2 diabetes and heart disease. Metformin is clinically proven to alleviate symptoms of diabetes, improving insulin sensitivity, spurring the conversion of sugar to energy and preventing the buildup of sugar in the liver. In the treated mice, $0.1 \%$ metformin appeared to burn more fat for energy, keep the mice's body weight low, increase the animals' antioxidant responses to cell stress, reduce inflammation and prevent the onset of metabolic syndrome.

The study's findings suggest that metformin may mimic some of the previously reported health benefits of calorie restriction, or limiting the amount of calories taken in each day from food. In this study, the diets of the mice were not calorie-restricted. In fact, treated mice ate

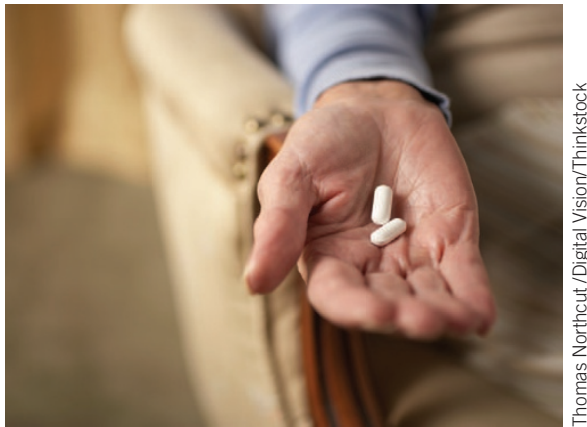

more calories than the untreated mice. This suggests that the drug may provide the benefits of calorie restriction without requiring food deprivation.

Studies in humans will have to confirm the results seen in these mice. The researchers point out that the lower dose of metformin used in the study led to blood levels in the mice that were 10 times higher than those in human patients treated with the drug for diabetes.

\section{Kara Rosania}

\section{NEW MODELS FOR STUDYING HEPATITIS C}

For decades, chimpanzees have been the primary animal model for studying hepatitis C virus (HCV) infection. Efforts to develop other animal models have so far failed because chimps are the only species other than humans that are naturally susceptible to HCV. But as regulations surrounding chimpanzee research in the US have become more restrictive, the need for alternative models to test potential drugs and vaccines against HCV has become more urgent. Now, two independent research groups report advances in the development of new animal models for studying HCV, one rodent and the other primate.

While at The Rockefeller University (New York, NY), Alexander Ploss began developing a mouse model of HCV infection by engineering mice to express two proteins found on the outside of human liver cells, CD81 and occludin. This enabled the virus to infect the animals but not to replicate well. So Ploss's team took it a step further, breeding the liver-protein-expressing mice with another mouse line engineered to have a defect in antiviral response. The offspring of this cross are susceptible to entry and replication of HCV (Nature published online 31 July 2013; doi:10.1038/nature12427). The team hopes that the mice will prove useful for studying the HCV infection cycle in vivo, testing candidate drugs and evaluating vaccine efficacy. "It has been very difficult to get to this point," Ploss told Nature News. Although "this model still cannot replace chimpanzees," he said, "it gives us a first glimpse of what may be possible with mice in coming years."

Meanwhile, across town at Icahn School of Medicine at Mount Sinai (New York, NY), Matthew Evans and Valerie Gouon-Evans were working to create a more closely related model for HCV infection in pigtail macaques (Macaca nemestrina). Liver cells that had been differentiated from macaque stem cells supported the HCV life cycle in vitro, although HCV infection efficiency was lower than in human liver cells. By modifying either of the same two liver proteins that Ploss's team altered in mice, CD81 and occludin, Evans' group increased HCV infection efficiency of the macaque cells (Gastroenterol. published online 26 July 2013; doi:10.1053/ j.gastro.2013.07.026). If in vivo experiments are successful, the research may lead to the validation of the pigtail macaque as a model for HCV studies and vaccine development. "These findings may open doors for the field of HCV research, lead to new animal models, and hopefully vaccines and therapies," Evans said in a press release.

Monica Harrington 